\title{
A laboratory-based nonlinear dynamics course for science and engineering students
}

\author{
N. Sungar, ${ }^{\text {a) }}$ J. P. Sharpe, M. J. Moelter, and N. Fleishon \\ Physics Department, California Polytechnic State University, San Luis Obispo, California 93407 \\ K. Morrison and J. McDill \\ Mathematics Department, California Polytechnic State University, San Luis Obispo, California 93407 \\ R. Schoonover \\ Chemistry Department, California Polytechnic State University, San Luis Obispo, California 93407
}

\begin{abstract}
We describe the implementation of a new laboratory-based interdisciplinary undergraduate course on nonlinear dynamical systems. Geometrical methods and data visualization techniques are especially emphasized. A novel feature of the course is a required laboratory where the students analyze the behavior of a number of dynamical systems. Most of the laboratory experiments can be economically implemented using equipment available in many introductory physics microcomputer-based laboratories.
\end{abstract}

\section{INTRODUCTION}

Over the last few decades there has been great interest in the area of dynamical systems, especially those involving nonlinearities. There are now several introductory textbooks available (e.g., Refs. 1 and 2). A comprehensive list of activities in the field of nonlinear dynamics along with references may be found in a recent AJP Resource Letter. ${ }^{3}$ A characteristic of the modern approach to teaching dynamical systems has been the use of geometrical methods and computer simulations. While there are many graduate-level courses in nonlinear dynamics, these methods do not yet appear to have been integrated into the typical undergraduate education of scientists and engineers. Traditionally, science and engineering students take a differential equations course, focusing on analytical solutions. Numerical methods, if discussed at all, take a secondary role. Students starting graduate school or in the workplace may, however, encounter systems that are too complicated to solve analytically. Although they can use the computer for numerical solutions, they have not developed any intuition about the typical behavior of such systems or the necessary skills to judge the validity of their computer-generated results. Examples of such systems include the behavior of lasers ${ }^{4}$ and biological population dynamics. ${ }^{5}$ To address this deficiency we have developed an upper-division, interdisciplinary, laboratory-based course that is currently offered as an elective to all science and engineering majors at Cal Poly. The objectives of this course are (i) to teach modern geometrical methods for analyzing differential equations and (ii) to demonstrate the use of visualization tools for data analysis. Geometrical concepts such as phase space, fixed points, attractors, bifurcations, limit cycles, and data analysis techniques such as power spectra, Poincaré sections, and return maps are emphasized. A key component of the course is a weekly laboratory where the students experiment with a number of often quite simple dynamical systems and learn to recognize the similarities and differences between seemingly disparate systems that are governed by similar equations. The lecture problems and experiments are chosen from a variety of disciplines including mathematics, chemistry, physics, and engineering.

\section{COURSE OUTLINE}

This course has a prerequisite of one year of calculus and a junior-level course in the student's major. We have included the requirement for a junior-level course to increase the chances for the student to have had exposure to dynamical systems within his/her own discipline. Each week there are three 1-h lectures and a 3-h lab. The lab consists of a variety of experiments ranging from those that demonstrate the similarities between the behavior of different physical systems to those that teach data analysis techniques. In our ten-week quarter students are given seven weeks of prescribed experiments and are required to do a project over the last three weeks. The students have a choice of either an advanced version of an experiment done in lab or an exploration of a physical system related to their major. We encourage the students to work on open-ended problems for their project.

The text for the course is Nonlinear Dynamics and Chaos. ${ }^{1}$ The book is at an appropriate level for juniors and seniors and uses many examples from a variety of disciplines. This excellent text enables students to understand the fundamental ideas very quickly and students are usually surprised to see how much information they can extract from the differential equations without having to solve them analytically.

Both the lectures and the labs are conducted in a studio classroom equipped with computers and a display system which allows the instructors to illustrate their lectures using software such as DIFFERENTIAL SYSTEMS ${ }^{6}$ and MATLAB ${ }^{7}$ on a daily basis. The students are regularly assigned homework problems, some of which require the use of computers. Several times during the quarter students are assigned a group problem to work in teams of two or three during the class time. During the exams, students have access to computers and the same software used in class and for homework.

\section{COURSE CONTENT}

The course follows the textbook fairly closely, starting with the notion of fixed points and phase plots, proceeding through bifurcations and on to multidimensional systems. 
Table I. Nonlinear dynamics course outline.

\begin{tabular}{|c|c|c|}
\hline Topic & Emphasis & Time spent \\
\hline $\begin{array}{l}\text { One-dimensional systems, } \\
\text { linear and nonlinear }\end{array}$ & $\begin{array}{l}\text { Basic concepts such as phase } \\
\text { diagrams and fixed points and } \\
\text { the terminology involved }\end{array}$ & 2 weeks \\
\hline Bifurcations & $\begin{array}{l}\text { Parameter space, different types } \\
\text { of bifurcations in one- } \\
\text { dimensional systems }\end{array}$ & 1 week \\
\hline $\begin{array}{l}\text { Two-dimensional linear } \\
\text { systems }\end{array}$ & $\begin{array}{l}\text { Eigenvalues and eigenvectors } \\
\text { and their representation in the } \\
\text { phase plane }\end{array}$ & 1 week \\
\hline $\begin{array}{l}\text { Nonlinear systems in } \\
\text { two or more dimensions }\end{array}$ & $\begin{array}{l}\text { Linearization around the fixed } \\
\text { point, topological properties of } \\
\text { the phase space for conservative } \\
\text { systems and reversible systems, } \\
\text { limit cycles }\end{array}$ & 2-3 weeks \\
\hline One-dimensional maps & $\begin{array}{l}\text { Systems with discrete time steps, } \\
\text { return maps }\end{array}$ & 1 week \\
\hline $\begin{array}{l}\text { Bifurcations in two- } \\
\text { dimensional systems }\end{array}$ & $\begin{array}{l}\text { Expansion of the concept of } \\
\text { bifurcation to two dimensions, } \\
\text { Hopf bifurcations and Poincare } \\
\text { maps }\end{array}$ & $1-2$ weeks \\
\hline Lorenz equation & $\begin{array}{l}\text { Introduction to strange attractors } \\
\text { and chaos, exploration of the } \\
\text { parameter space }\end{array}$ & 1 week \\
\hline
\end{tabular}

Discrete systems are considered. The course culminates with a brief introduction to chaos. Table I shows the general course content, the emphasis for each topic, and time spent on each topic.

\section{LABORATORY}

The laboratory component of the course meets for one 3-h period each week. We have at most eight students per lab section, working in groups of two. Students are asked to write a lab report for each experiment and the lab reports count for $20 \%$ of their overall grade. One out of five questions on each exam was directly related to the experiments. In the studio classroom, which is used for both lectures and labs, the students have access to Macintosh computers. As well as hosting DIFFERENTIAL SYSTEMS and MATLAB, the computers are used for data collection and analysis. Data collection is accomplished with a variety of probes (e.g., motion, temperature, voltage, current) interfaced through Universal Laboratory Interface (ULI). Software for use with this microcomputer-based laboratory system allows data display and preliminary analysis. ${ }^{8}$

The first seven weeks are devoted to the experiments that are described below and summarized in Table II. The last three weeks are used for a required project which counts for $10 \%$ of the overall grade. The experiments were developed by a group of faculty from the physics, chemistry, and math departments. Some of the experiments are adaptations of published experiments for which the references are included in the descriptions below. We have a more complete write-up for each of these experiments available on our web page (http://www.calpoly.edu/ nsungar/experiments.html).

\section{DESCRIPTION OF THE EXPERIMENTS}

\section{A. Numerical methods}

This is a simple exercise that demonstrates the importance of the step size in using the Euler method and familiarizes students with the software. Students are given a simple onedimensional system and asked to integrate first without a computer using several different step sizes and compare with the analytical solution. They then progress to doing the integration using an Excel spreadsheet and then MATLAB and DIFFERENTIAL SYSTEMS. This exercise takes approximately $1 \mathrm{~h}$.

\section{B. One-dimensional systems}

The purpose of these four experiments is to show the similarities and differences between several one-dimensional systems and to demonstrate the idea of fixed points. The first part consists of two linear systems that have the same dynamical behavior: (i) Newton's law of cooling-a temperature probe monitors the temperature change for an object that is cooler (and later warmer) than the ambient temperature. Students can measure the temperature versus time ( $T$ vs $t$ ) and phase portrait $(\dot{T}$ vs $T)$ in real time and see how the system always moves toward a fixed point independent of the initial conditions. (ii) $\mathrm{RC}$ circuit - the discharging of a $\mathrm{RC}$ circuit is monitored with voltage and current probes. Again, the phase plot is observed by plotting the current versus the voltage across the capacitor (i.e., $\dot{q}$ vs $q$ ).

Following the linear systems, students work on two nonlinear systems. (iii) Titration-in a titration experiment, a $p \mathrm{H}$ probe monitors the $p \mathrm{H}$ as acid is added at a constant rate to a base. A real time plot of $p \mathrm{H}$ vs $t$ and the phase plot $p \dot{H}$ vs $p \mathrm{H}$ are produced. (iv) Terminal velocity - in a terminal velocity experiment a motion detector monitors the motion of a "frictionless" cart on a track, pulled by a small spherical mass attached to a string that hangs over a pulley and falls inside a long tube filled with a viscous fluid (we found liquid detergent to be the most convenient). The dynamical variable in this experiment is the velocity, and students are asked to find the functional dependence of the drag force on velocity from their data (linear at low velocities with a quadratic term coming in at higher speeds).

All of these experiments are more or less qualitative, and the analysis of the results involves answering a series of questions pertaining to the graphs obtained. Representative data from some of these experiments are shown in Fig. 1. The experiments are spread over a two-week period, with the terminal velocity experiment taking the most time to perform.

\section{Bead on a rotating hoop}

This experiment was included to introduce the idea of bifurcations and is based on Sec. 3.5 in the textbook. ${ }^{1}$ While several articles in the literature have drawn attention to the bifurcation aspects of this experiment, ${ }^{9,10}$ none obtain quantitative data. A simple mechanical system, consisting of a slice of 6-in.-diam PVC pipe that is mounted on a variable speed motor, is used (Fig. 2). Two wire tracks on the inside surface hold a small steel ball that is free to roll on the tracks. A brief video recording of the system is made at each rotation frequency once the ball reaches its fixed point and is steady. These recordings are analyzed by using the "freeze- 

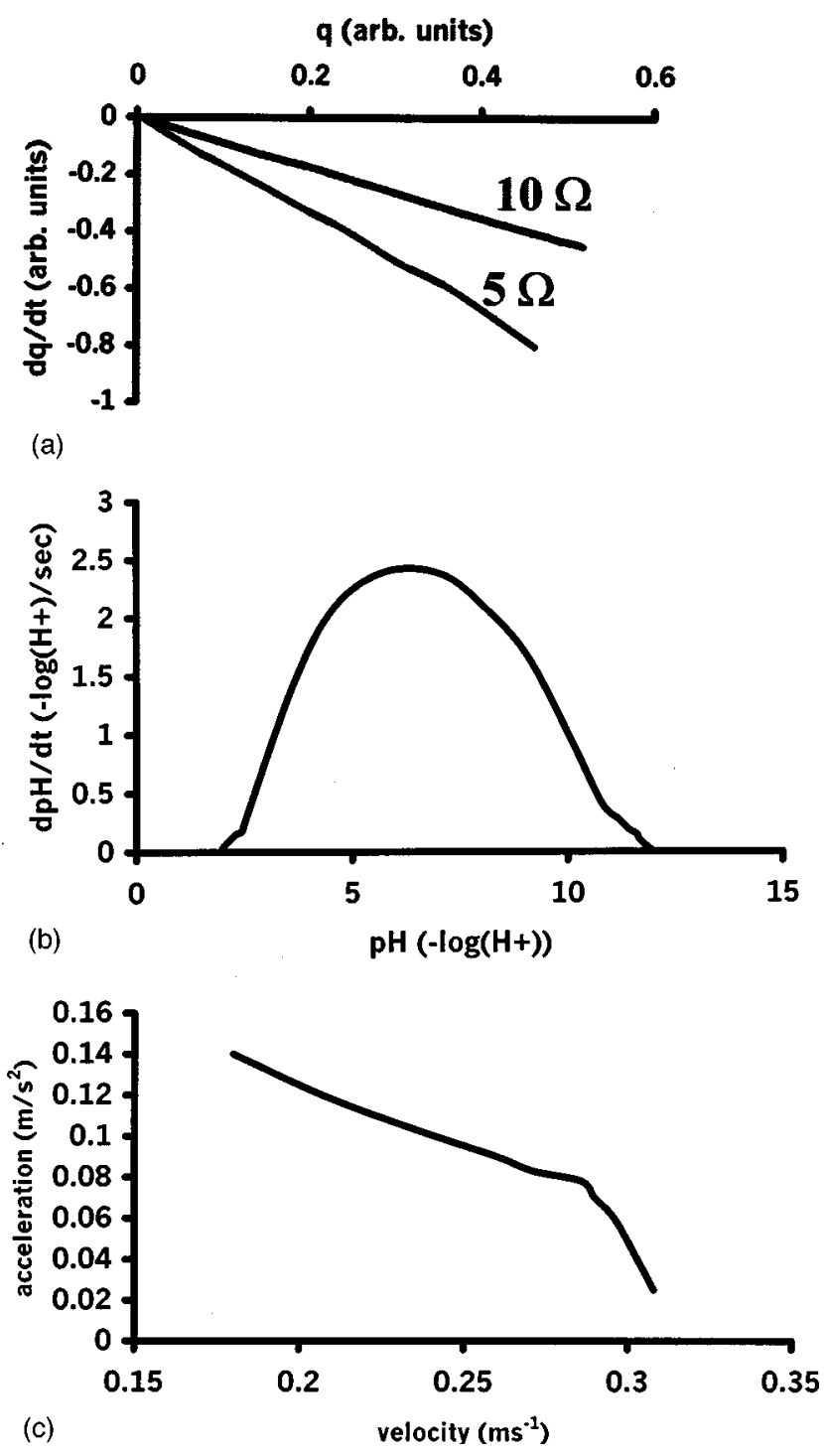

Fig. 1. Data from some simple one-dimensional systems experiments. (a) $\dot{q}$ vs $q$ in the RC experiment where the upper, line shows the result of using a $10-\Omega$ resistor and the lower line shows the result of a $5-\Omega$ resistor. (b) $p H$ vs $p \mathrm{H}$ for the titration experiment. (c) $\dot{v}$ vs $v$ for the terminal velocity experiment. Note that cases (b) and (c) are nonlinear.

frame" capability of the video recorder to determine the stable angular position of the ball for each frequency. Students plot the stable angle versus the rotation frequency diagram and compare it with the theoretical graph (see Fig. 3). Our particular implementation of the experiment can also crudely show the effect of imperfections on bifurcations by simply offsetting the rotation axis from vertical. However, this aspect is not quantitatively pursued as it is rather time consuming to extract data using the video recorder. This experiment takes approximately $2 \mathrm{~h}$ to perform.

\section{Two-dimensional systems}

A vertically mounted mass-spring system and a RLC circuit are used to demonstrate the behavior of linear twodimensional systems and to show how oscillations are represented in the phase plane. The mass-spring system is monitored with a motion detector and the evolution of the system in the phase plane ( $\dot{x}$ vs $x$ ) is observed in real time. Damping is varied by attaching a piece of cardboard to the
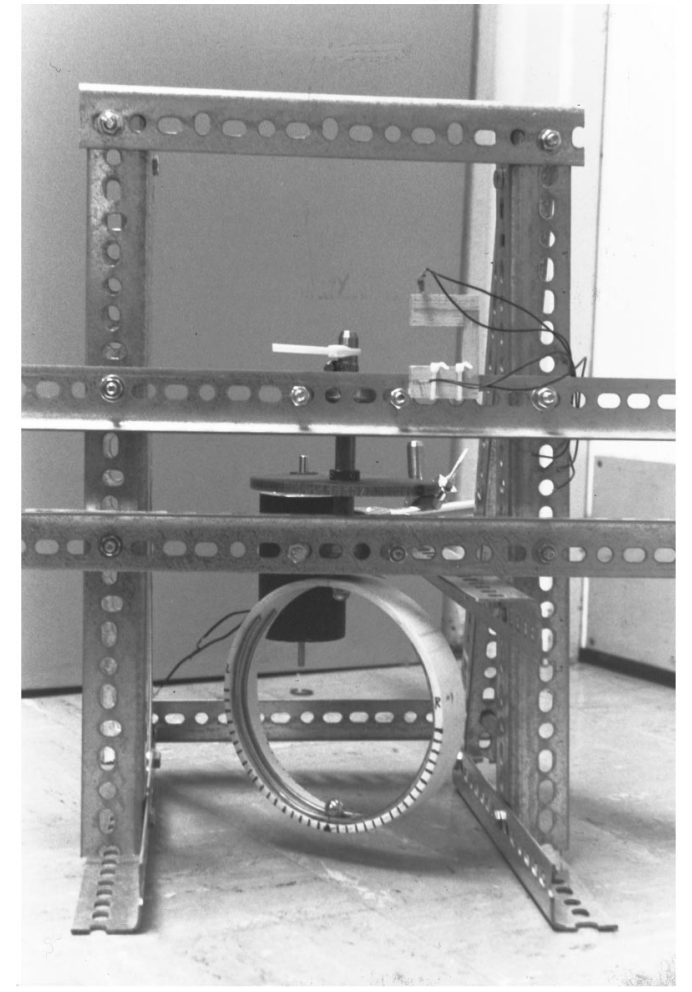

Fig. 2. Photograph of the bead on a hoop apparatus. The hoop is constructed from 6-in.-diam PVC pipe with brass rails on the interior to hold the steel ball. The hoop is suspended through two radial bearings using a half-inch diameter metal post and the post connected to the motor (visible at the back) by gears and a toothed plastic belt (available from Berg Manufacturing). Connected to the middle horizontal member is a photogate used to measure the rotation frequency. Power supply and frequency meter are not pictured.

mass. The mass and the initial conditions are also varied. The RLC circuit is also analyzed to demonstrate the similar behavior of systems that are governed by the same differential equations. We monitor the voltages across the resistor and the capacitor using an oscilloscope while we vary the parameters by using variable resistors and inductors. Similarities and differences between the two experiments are discussed.

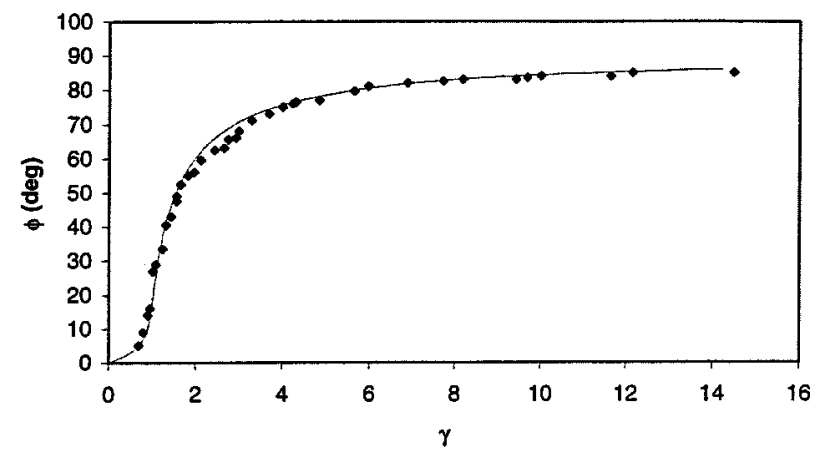

Fig. 3. Results from the bead on a hoop experiment (points). The solid line is a plot of a theoretically expected angular displacement $(\phi)$ vs the parameter $\gamma=r \omega^{2} / g$ for a hoop with the center of rotation slightly displaced from the radius of the hoop. $r$ is the hoop radius, $\omega$ is the angular velocity, and $g$ the acceleration due to gravity. Note that the experimental data do not directly approach $(0,0)$, showing a slight imperfection in the system. 


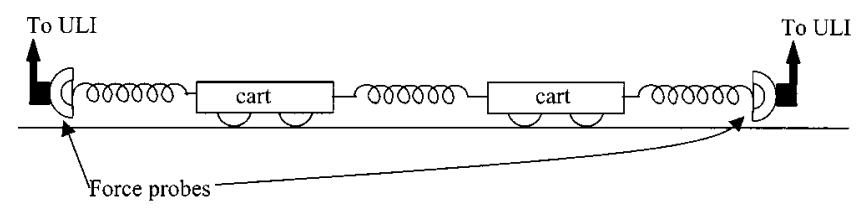

Fig. 4. Schematic of the coupled oscillator experiment.

\section{E. Multidimensional systems}

Two identical "frictionless" carts are connected with identical springs to produce a coupled mass-spring system (Fig. 4). This four-dimensional linear system demonstrates normal modes and their relationship to the concepts of eigenvalues, eigenvectors, and diagonalization of the system matrix. The motion of the system is monitored by attaching the outer springs to computer interfaced force probes. We keep the displacements small enough so that the proportionality between force and position can be used to monitor the positions of both carts simultaneously. (The reason for using force probes instead of motion detectors is the difficulty in simultaneously using two facing ultrasonic motion detectors.) The students are asked first to start the system in each one of the normal modes and then in an arbitrary mode of oscillation. They observe the sum and the difference of the positions of the two carts to see how the normal modes relate to a change of variables and hence the diagonalization of the four-dimensional system matrix which reduces the system to two, uncoupled, two-dimensional systems. They also use the fast Fourier transform tool on the DATA LOGGER software to see the frequencies present in the motion of the carts, one for each normal mode, demonstrating that any arbitrary motion consists of a combination of only these frequencies. This experiment takes roughly $2 \mathrm{~h}$ to complete.

\section{F. Ship stability}

This is a computer simulation experiment using a simplified model of a ship in rolling seas in order to study the ship's stability and the conditions for capsizing. ${ }^{11}$ This experiment is designed to demonstrate that, although a ship in the sea is a very complicated system, one can model it reasonably effectively using a simple model and that, even though the model is simple, the behavior is not. The model is a forced, damped, nonlinear oscillator. Students analyze this system using DIFFERENTIAL SYSTEMS and MATLAB. They start with no damping and forcing and progressively add the damping and the forcing terms. They produce a stability diagram showing the regions of stability and inevitable capsizing as the forcing amplitude and forcing frequency are varied. The concept of an effective potential energy is also emphasized in this experiment. This experiment takes $3 \mathrm{~h}$.

\section{G. Analysis of a complex nonlinear oscillator}

Techniques for display and analysis of data generated by the motion of a nonlinear mechanical system are investigated. The experiment is derived from Ref. 12. A permanent magnet is mounted on a rotation probe that is interfaced to the computer and inserted at the center of a set of Helmholtz coils driven by a sinusoidally varying current [Fig. 5(a)]. The
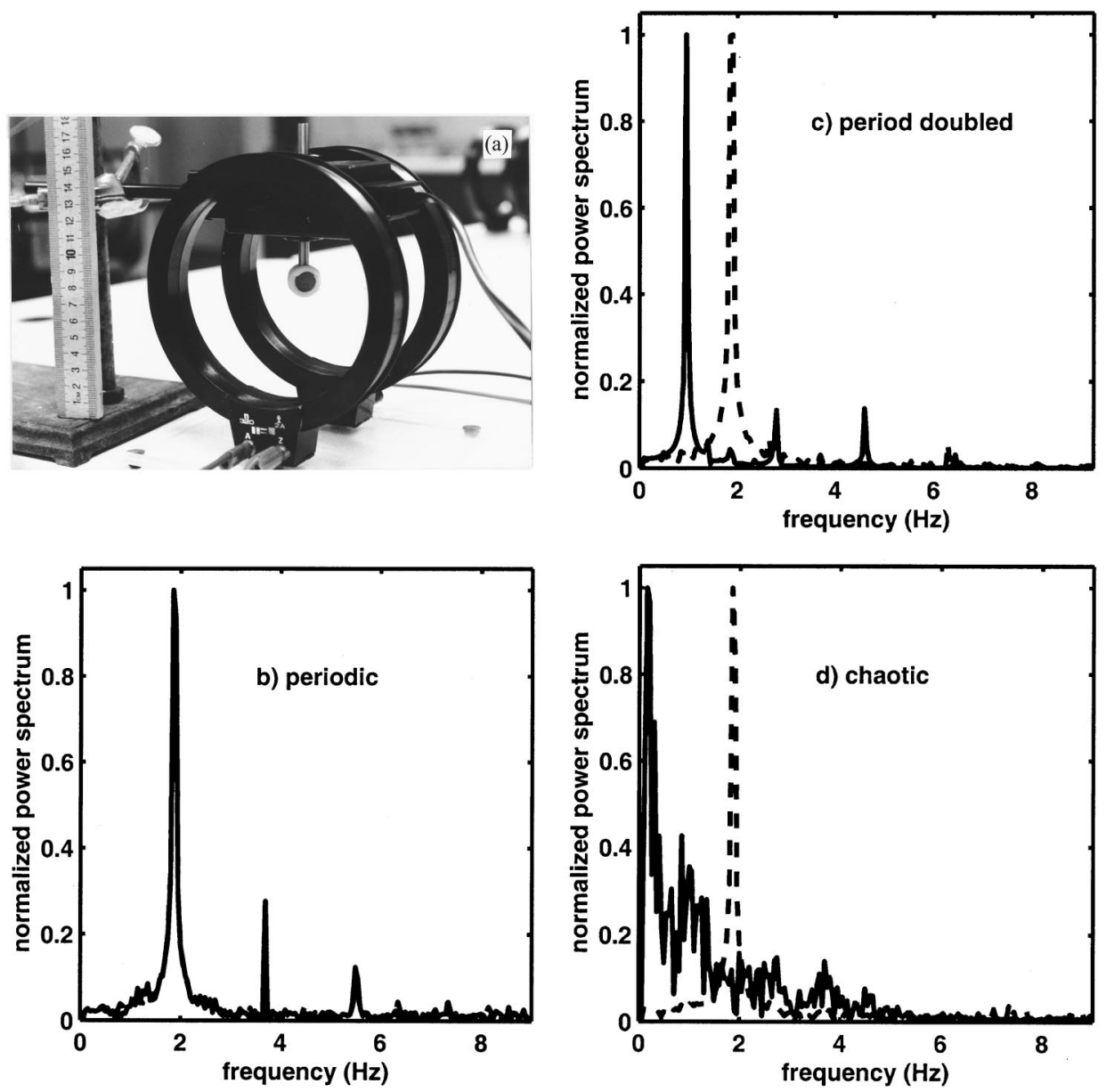

Fig. 5. (a) Photograph of the rotating magnet apparatus. (b), (c), and (d) show the result of the Fourier transform of the output when the motion is periodic, period doubled, and chaotic, respectively (solid curves). The dotted curves show the power spectrum of the driver at $1.8 \mathrm{~Hz}$. 
current through the coils, which is proportional to the forcing term in the governing equation, is monitored using a current probe that is also interfaced to the computer. This system shows an array of complex, though structured, behaviors which range from simple periodic to period doubled to chaotic. Although an equation modeling an idealized version of this system can be written down, the primary purpose in this experiment is not to explain the observed behavior from first principles. Real systems are, in general, too complex to be modeled with great accuracy. The purpose is to discover useful ways of analyzing and interpreting the data in order to identify signatures of different types of behavior. The techniques we use include graphs of Fourier spectrum analysis, phase space trajectories, and Poincaré sections. Figure 5 shows samples of the power spectra of the velocity-time history of the magnet for periodic, period doubled, and chaotic motion.

\section{H. Dripping faucet}

The purpose of this experiment is to demonstrate the techniques used in the analysis of discrete systems. Water dripping from a faucet displays many features of nonlinear systems including chaotic transitions, bifurcations, and multiple basins of attraction as the flow rate is varied. ${ }^{13}$ In this experiment the time interval between water drops from a dropper at different flow rates is measured. The water is kept at a constant head of pressure using a weir cup that is supplied by a simple fish tank pump (available at pet stores) and the flow rate varied using a clamp. A photograph of the apparatus is shown in Fig. 6(a). It was found that it was easiest to generate interesting regimes of behavior using a dropper with a fairly wide exit hole ( $>2 \mathrm{~mm}$ i.d.). The experiment is simple to set up using a photogate (Vernier VPG-DG) that is interfaced to the computer through the ULI box using the software ULITIMER. The students then use a spreadsheet to generate return maps and histograms to look for signatures of different types of behavior [Fig. 6(b)]. This experiment takes $3 \mathrm{~h}$.

\section{OTHER EXPERIMENTS}

In addition to these experiments we had developed two others that were included the first time we taught the course but taken out later. These are the inverted pendulum and oscillating chemical reaction experiments.

The inverted pendulum is a simple mechanical system exhibiting complex nonlinear behavior ${ }^{14}$ and exhibits a range of behavior comparable to the spinning magnet experiment. The students had a choice between the spinning magnet and the inverted pendulum experiments. Both used the same data analysis techniques. However, we found that a simple implementation of the inverted pendulum experiment using a spring-driven hacksaw blade as the pendulum was not suitable for a 3-h lab period. The system was very sensitive to initial conditions with very long transients. This made finding the regimes of different behavior quite challenging. Also, since the regimes of interesting behavior occurred when the blade was almost horizontal it was very difficult to use the ultrasonic motion detectors. A much more sophisticated implementation of the experiment can be found in Ref. 14, where great care is taken to isolate the system from surrounding perturbations like air currents and the motion is monitored using strain gauges attached to the blade. Since
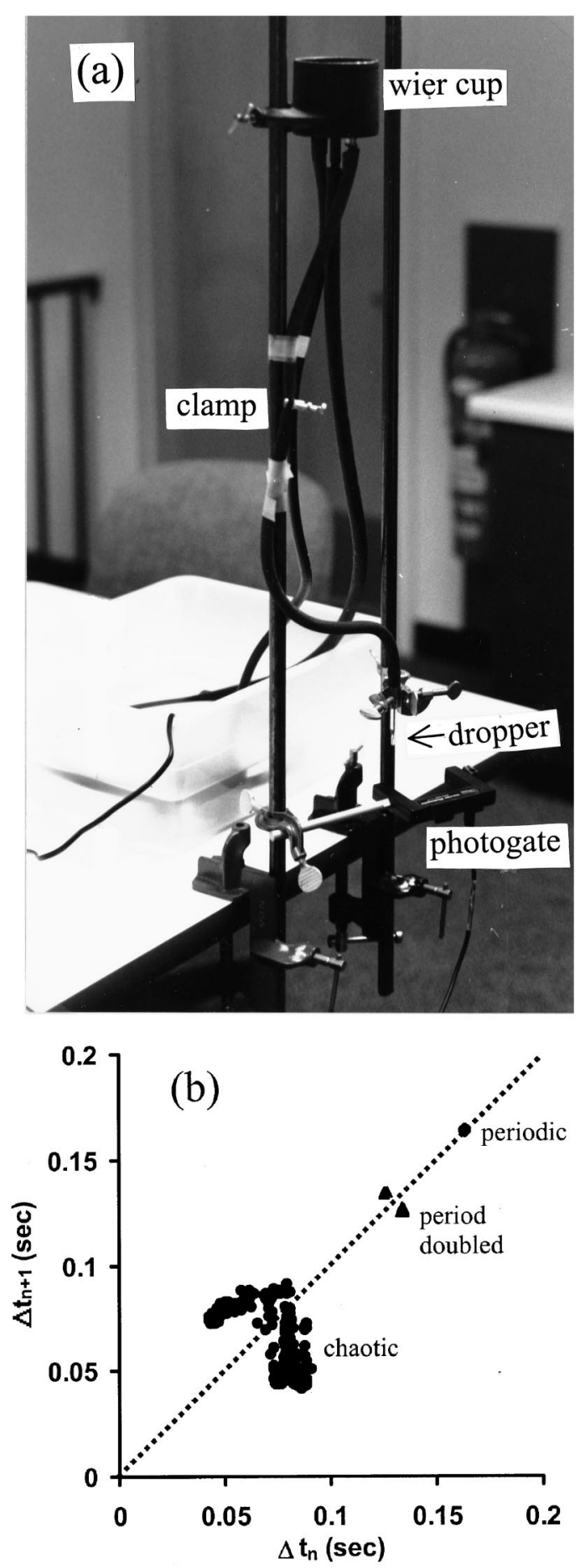

Fig. 6. (a) Photograph of the dripping faucet apparatus. (b) Return maps generated from the dripping faucet experiment showing, at different flow rates, the appearance of periodic, period doubled, and chaotic behavior.

the spinning magnet experiment gave good results quite readily, we decided to eliminate the inverted pendulum experiment and offer it as a project instead.

An oscillating chemical reaction experiment ${ }^{15}$ was originally part of multidimensional systems and was designed to show oscillatory behavior in chemical reactions. In the execution of the experiment, we encountered a lot of difficulty with data acquisition. Inert sampling electrodes were used to simultaneously monitor the bromide concentration and con- 
Table II. Schedule of prescribed experiments for nonlinear dynamics course laboratory.

\begin{tabular}{|c|c|}
\hline Experiment & Involves \\
\hline Numerical methods & $\begin{array}{l}\text { Introduction to computers and software. } \\
\text { Numerical integration (first week) }\end{array}$ \\
\hline One-dimensional systems & $\begin{array}{l}\text { Newton's law of cooling, RC circuit, titration, } \\
\text { terminal velocity (first and second weeks) }\end{array}$ \\
\hline Bead on a rotating hoop & $\begin{array}{l}\text { Demonstration of a bifurcation } \\
\text { (second and third weeks) }\end{array}$ \\
\hline Two-dimensional systems & $\begin{array}{l}\text { Mass-spring, RLC circuit } \\
\text { (third and fourth weeks) }\end{array}$ \\
\hline Multidimensional systems & Coupled oscillators (fourth week) \\
\hline Ship stability & Computer experiment (fifth week) \\
\hline Nonlinear oscillator & $\begin{array}{l}\text { A spinning magnet in a sinusoidally } \\
\text { varying magnetic field (sixth week) }\end{array}$ \\
\hline Discrete systems & Dripping faucet (seventh week) \\
\hline
\end{tabular}

duction of an oscillating system. However, the ion-selective electrodes were delicate and leaked quite easily, ruining the reactions. Students were able to visually observe the oscillation in the color of the solution; however, none of the groups were able to collect data for quantitative analysis. We decided to eliminate this experiment due to both the difficulties involved and the time constraints.

\section{PROJECT}

Over the last three weeks of the quarter the students worked on a project either in a team of two or individually. They were given a list of suggestions, but were free to choose a project that was feasible in the time frame allowed and with the existing equipment. They were also encouraged to talk to other faculty for ideas, especially for projects related to their major. Another possibility was to perform a more advanced version of one of the experiments they had done previously. Most of the students found this aspect of the course very enjoyable and an excellent learning experience. The problems they chose were mostly open ended and they were required to plan, perform, and report their exploration with minimal supervision. The following list indicates some of the more interesting projects undertaken by the students with the student's major indicated in parentheses:

- Building a chaotic waterwheel (Physics)

- Observing bifurcations in a buckling beam (Civil Engineering) ${ }^{1}$

- Coupled pendulums (Physics) $)^{16,17}$

- The Mercury Beating Heart (Chemistry) ${ }^{18}$

- Construction of Chua's chaotic circuit (Electrical Engineering) ${ }^{19}$

- Observation of pattern formation in heated liquids $(\text { Math. })^{20}$

- Refined analysis of the bead on a hoop experiment (Aero. Engineering)

It may be interesting to note that most of these experiments were undertaken using the facilities of an introductory studio lab, supplemented with supplies from the local hardware stores. In only a few instances did we have to borrow from the upper-division labs for items like digitizing oscilloscopes and frequency meters.

\section{DISCUSSION}

The course has now been offered twice, with the lectures and labs being taught by Math and Physics faculty. It is also clear from the experiments done in the course and the projects undertaken that the weighting is toward physics. In the future we hope to induce biological and engineering sciences faculty to participate and offer examples from those areas. It may be interesting to note that the second time the class was offered, almost half of the students were engineering majors and that several of these students found direct application of the course techniques in their senior and masters projects.

As might be expected given the prerequisites for the class, there were large differences in the background preparation among the students and this led to the risk of boring some and perplexing others. However, we found that the techniques we were introducing were sufficiently new to keep most of the class stimulated. Also, in the laboratories, the instructors were mindful of these background differences and ensured, for example, that students from biological sciences were not working on final projects that needed a detailed knowledge of Fourier techniques.

Overall the course seems to have been successful in its objectives and has received a very positive response from the students. We conducted extensive surveys of the students at the end of each year, the results of which can be summarized as follows

- The majority of the students strongly agreed that the experiments helped them understand the material. We found that there was some reluctance from the math majors who were unhappy about working with equipment which was unfamiliar to them and often felt they did not need the lab work.

- All the students agreed that this course changed the way they think about dynamical systems and differential equations and those who had already taken a differential equations course were surprised at the amount of information that could be extracted from the equations using qualitative arguments.

- The students found the difficulty level of the course less than they had anticipated.

- The majority of the students found the project part of the course very enjoyable and chose a topic in their own field of interest.

- The majority of the students indicated that they would have liked to spend more time on the topic of chaos.

\section{ACKNOWLEDGMENT}

This work was supported by NSF Grant No. DUE9554909.

\footnotetext{
${ }^{a)}$ Electronic mail: nsungar@calpoly.edu

${ }^{1}$ S. H. Strogatz, Nonlinear Dynamics and Chaos: with Applications to Physics, Biology, Chemistry, and Engineering (Addison-Wesley, Reading, MA, 1994).

${ }^{2}$ R. C. Hilborn, Chaos and Nonlinear Dynamics: An Introduction for Scientists and Engineers (Oxford U.P., New York, 1994).

${ }^{3}$ Robert C. Hilborn and Nicholas B. Tufillaro, "Resource Letter ND-1: Nonlinear Dynamics," Am. J. Phys. 65, 822-834 (1997).

${ }^{4}$ W. Lauterborn, T. Kurz, and M. Wiesenfeldt, Coherent Optics: Fundamentals and Applications (Springer, New York, 1995), Chap. 10.

${ }^{5}$ J. D. Murray, Mathematical Biology (Springer Verlag, New York, 1993).

${ }^{6} \mathrm{H}$. Gollwitzer, DIfFerential systems 4.0 (U'Betcha Publications, 554
} 
Evans Road, Springfield, PA 19064, 1996); (610) 544-9257, hgollwit@mcs.drexel.edu

${ }^{7}$ MATLAB 5.2 available from The Mathworks, Inc., 24 Prime Park Way, Natick, MA 01760; (508) 647-7000, http://www.mathworks.com

${ }^{8}$ The Universal Laboratory Interface (ULI) boxes are available from Vernier Software and Technology (www.vernier.com). Tel: (503) 277-2299. This company also supplies probes for temperature, current and voltage, force, motion (both linear and rotational) and $p \mathrm{H}$. Data transfer was via a number of Vernier software packages including DATALOGGER, MACMOTION, ELECTRICITY, and ULITIMER.

${ }^{9} \mathrm{G}$. Fletcher, "A mechanical model of first- and second-order phase transitions," Am. J. Phys. 65, 74-81 (1997).

${ }^{10} \mathrm{R}$. V. Mancuso, "A working mechanical model for the first- and secondorder phase transitions and the cusp catastrophe," Am. J. Phys. 68, 271277 (2000).

${ }^{11}$ S. R. Bishop and M. S. Soliman, "The prediction of ship capsize: Not all fractals are environment friendly," in Application of Fractals and Chaos, edited by Crilly, Earnshaw, and Jones (Springer-Verlag, New York, 1993).

${ }^{12} \mathrm{~K}$. Briggs, "Simple experiments in chaotic dynamics," Am. J. Phys. 55, 1083-1089 (1987).
${ }^{13} \mathrm{~K}$. Dreyer and F. R. Hickey, "The route to chaos in a dripping water faucet," Am. J. Phys. 59, 619-627 (1991).

${ }^{14}$ J. E. Berger and G. Nunes, "A mechanical Duffing oscillator for the undergraduate laboratory," Am. J. Phys. 65, 841-846 (1997).

${ }^{15}$ Earle S. Scott, Rodney Schreiner, Lee R. Sharpe, Bassam Z. Shakhashiri, and Glen E. Dirreen, "Oscillating Chemical Reactions," in Chemical Demonstrations, edited by B. Z. Shakhashiri (University of Wisconsin Press, Madison, WI, 1985), Vol. 2, Chap. 7.

${ }^{16}$ T. Shinbrot, C. Grebogi, J. Wisdom, and J. A. Yorke, "Chaos in a double pendulum," Am. J. Phys. 60, 491-499 (1992).

${ }^{17}$ R. B. Levien and S. M. Tan, "Double pendulum: An experiment in chaos," Am. J. Phys. 61, 1038-1044 (1993).

${ }^{18}$ J. A. Campbell, "Beating Heart," J. Chem. Educ. 34, A105 (1957), and also see the letter on p. 362 .

${ }^{19}$ M. P. Kennedy, “Three Steps to Chaos. I Evolution,” IEEE Trans. Circuits Syst., I: Fundam. Theory Appl. 40 (10), 640-656 (1993); “Three Steps to Chaos. II A Chua's Circuit Primer,' 40, 657-674 (1993).

${ }^{20}$ S. J. Van Hook and M. F. Schatz, "Simple demonstrations of patternformation,"' Phys. Teach. 35, 391-395 (1997). 